\title{
Dynamic Control-limit Policy of Condition based Maintenance for the Hydroelectricity Generating Unit
}

\author{
Xinbo Qian ${ }^{1}$ and Yonggang $\mathrm{Wu}^{2}$ \\ ${ }^{1}$ School of Hydropower and Information Engineering, \\ Huazhong University of Science and Technology \\ 1037 Luoyu Road, China, 430074 \\ ${ }^{2}$ School of Hydropower and Information Engineering, \\ Huazhong University of Science and Technology \\ 1037 Luoyu Road, China, 430074 \\ 'simble777@163.com, ${ }^{2}$ ygangwu@163.com
}

\begin{abstract}
This paper considers a condition-based maintenance (i.e. CBM) model for the hydro generating unit in the deregulated power system. As the generating loss varies according to the time-varying inflows, the economic dependence among the critical component of the generating unit is dynamic. To reduce the maintenance cost effectively, we propose an inflowdependent control-limit policy instead of the constant control-limit policy for CBM optimization. An example for the hydro generating unit is presented to verify the effectiveness of the proposed policy.
\end{abstract}

Keywords: Condition based maintenance, Optimization solution, Inflow, Proportional hazards model, Economic dependence

\section{Introduction}

The reliability of generating units is very important for the power system with high reliability [1-3]. Maintenance can be an efficient way to improve the reliability and extend the lifetime of the units. The maintenance strategies for generating unit can be divided into planning maintenance (i.e., maintenance scheduling) and condition based maintenance (i.e., CBM). The development of maintenance scheduling environment for generating units has been developed from the traditional centralized power system to the deregulated power systems. In a traditional centralized power system, the independent system operator (ISO) [4] schedules maintenance time for all generating units in a corresponding area, aiming to keep the reliability level of the whole power system, meanwhile reducing the total maintenance cost. A great number of papers have been published for the solution of such a problem, such as $[5,6]$.

Planning maintenance is a kind of maintenance strategy to improve the reliability of the generating unit. However, to improve the effectiveness of maintenance actions, condition based maintenance should be involved as well as the planning maintenance. For the research about CBM for generating unit, the generating unit was simplified as a single-component system; actually it is multi-component system in practice. The maintenance policy of a multicomponent system differs from that of a single-unit system because dependencies exist among components, including economic dependence, structural dependence and stochastic dependence [7]. In this research, we focus only on the economic dependence. Economic 
dependence means that performing maintenance on several subsystems jointly costs less money and/or time than on each subsystem separately [8]. Therefore, there is often a great potential for cost savings by implementing an opportunistic maintenance policy [9-11]. Opportunistic maintenance basically refers to the situation in which preventive maintenance is carried out at opportunities, either by choice or by restriction. For example, it is possible to do preventive maintenance for non-failed subsystems at a reduced additional cost while failed subsystems are being repaired. According to different criterions, the opportunistic maintenance for the multi-component system can be divided into three categories: traditional reliability approaches, condition data-based maintenance and integrated approaches.

Traditional reliability approaches can be referred to as event data based maintenance models, which can be divided into two main categories: time-based opportunity maintenance and failure rate-based opportunity maintenance. For time-based opportunity maintenance policy, upon a component failure the other component as well as the failed one is performed maintenance if its age exceeds a pre-determined control limit. More studies on time-based maintenance of multi-component systems have been reported in literature $[10,12]$. Zheng et al. [11] examined an opportunistic maintenance policy based on failure rate tolerance for a system with multi-type units. Attention is restricted to policies which take actions based on the actual condition of all components. The 'age' of a component or the failure rate is usually used as the condition variable. However, the aforementioned maintenance models did not consider the condition monitoring information, such as vibration data, acoustic emission data, oil analysis data and temperature data.

Current condition state can support the short-term maintenance strategy, instead of providing the indication of failure time or probability of failure [13]. To utilize the available information fully for a long-term maintenance strategy, the integrated approaches, which are based on both event and condition data, can be promising research challenges [13]. A PHM (i.e. proportional hazard model) based CBM policy for multi-component systems was proposed in [14], for which economic dependence exists among different components. Upon a component failure the other component as well as the failed one is performed maintenance if its hazard risk exceeds a pre-determined control-limit. While for cases with time-dependent economic dependences, there may exist the potential profit if assign different thresholds to different periods. For example, the generation losses can be time-dependent for the hydroelectricity generation unit since the inflow is time-varying.

To deal with the problem, we propose an inflow-dependent control-limit policy for CBM for the generating unit as a multi-component system. Comparative numerical examples for a hydro generating unit are presented to verify the effectiveness of the proposed CBM policy.

The rest of the paper is organized as follows. In Section 2, inflow-dependent control-limit policy for CBM optimization is proposed. Simulation studies are presented in Section 3. Finally, some conclusion remarks are given in Section 4.

\section{Inflow-dependent Control-limit Policy for CBM Optimization}

The valuable statistical procedure for estimating the risk of equipment failure when it is subjected to condition monitoring is the proportional hazard model (PHM) [15]. The forms of PHM combine the based hazard function $\mathrm{h} 0$ along with a component that takes into account covariates which are used to improve the prediction of failure. The particular form used in this study is known as a Weibull PHM which is a PHM with a Weibull baseline, and it is given by

$$
h(t, z(t))=h_{0}(a(t)) \exp (\gamma z(t))=\frac{\beta}{\eta}\left(\frac{a(t)}{\eta}\right)^{\beta-1} \exp (\gamma z(t)),
$$


where $\beta$ and $\eta$ are parameter of the proportional hazards model, $a(t)$ is the age of the component, $z(t)$ is the covariate value of the component at time $t$ and $\gamma$ is the corresponding coefficient of the covariate. The covariates, which can be considered as the key condition monitoring measurements reflecting the health condition of the equipment, can be obtained by the software EXAKT [16, 17]. The CBM optimization approach for the multi-component system based on proportional hazards model, and the method for calculating the cost and reliability objective function, were developed in Ref. [14]. For the PHM based CBM policy, two-level risk thresholds $\left\{x_{1}, x_{2}\right\}$ were introduced to determine which component should be performed preventive maintenance $(\mathrm{PM})$ or opportunistic maintenance $(\mathrm{OM})$ at a certain inspection point. The objective of the CBM optimization is to find the optimal thresholds $\left\{x_{1}, x_{2}\right\}$ to minimize the total maintenance cost.

Since the generation of the hydroelectricity generating unit varies according to the timedependent inflows. The outage cost of the generating unit varies according to the timedependent inflows, so the economic dependence among the critical components of the generating unit is dynamic. So some extended model should be studied for the dynamic cost structure [18]. In this section, we propose the inflow-dependent control-limit policy for CBM for the generating unit, considering the dynamic economic dependence among the components, as well as the reliability and the monitoring condition of different critical components.

The following assumptions are made in this paper regarding the multi-component systems under discussion:

(1) The generating unit is on operation continuously, unless it is shut down for maintenance.

(2) The components are repairable, and they are independent in their degradations and failure processes.

(3) We focus on the maintenance optimization in this study, and the inspection interval is not a design variable in the optimization problem. So the inspections are assumed to be performed for constant interval.

(4) The effect of maintenance is assumed to be as good as new for simplification.

In this work, we extend the CBM policy for multi-component systems from constant threshold to inflow-dependent threshold. The time-dependent inflow can cause the dynamic economic dependence among components. The proposed policy takes the dynamic economic dependence among components into consideration. The inflow-dependent two-level thresholds are introduced to determine which components should be preventively or opportunistically maintained. The time-dependent two-level risk thresholds $\left\{x_{1}(t), x_{2}(t)\right\}$ are characterized as follows:

$$
\begin{aligned}
& x_{1}(t)=I(t) \cdot y_{1} \\
& x_{2}(t)=I(t) \cdot y_{2}
\end{aligned}
$$

where $y_{1}$ and $y_{2}$ are the risk threshold scaling factors. $I(t)$ is the inflow index, and it can be calculated by $I(\mathrm{t})=R(t) / \bar{R}$, where $R(t)$ and $\bar{R}$ are the runoff of during time $\mathrm{t}$ and the mean runoff in a year, respectively. The inflow-dependent two-level risk thresholds are determined once the two risk threshold scaling factors $\left\{y_{1}, y_{2}\right\}$ are given. The inflow-dependent controllimit CBM policy extended from the constant-threshold CBM policy is proposed as follows: (1) Perform corrective maintenance if a failure occurs on component $i$. 
(2) For component i, preventive maintenance is performed if $K h>I(t) y_{1}$ where $K$ is the difference between the corrective maintenance and preventive maintenance.

(3) If preventive or corrective maintenance is performed on any other component of the system, perform opportunistic maintenance on component $i$ if $K h>I(\mathrm{t}) y_{2}$, where $y_{1}>y_{2}$.

For the proposed policy, two levels of inflow-dependent risk thresholds are used to deal with the time-varying economic dependence among different components, while inflowdependent two-level risk thresholds are used to determine whether preventive maintenance or opportunistic maintenance should be performed on a component.

For the inflow-dependent downtime cost, it can be cost efficient to utilize the timedependent risk threshold instead of the constant risk threshold. Qualitatively, for lower thresholds preventive maintenance can be performed with higher possibilities. Conversely, for higher maintenance thresholds, preventive maintenance can be performed with lower possibilities. As a result, the more the inflow the higher the thresholds are. So for the period with higher generation, the thresholds are higher; and the thresholds are lower during the period with lower generation. So it can be cost-effective to perform PM with lower (respectively, higher) possibility during periods of more (respectively, less) inflows.

The maintenance cost $C$ involves the corrective maintenance cost, preventive maintenance cost, opportunistic maintenance cost as well as the loss of generation during the maintenance. The maintenance cost $C$ during the planning horizon is given by

$$
C=\sum_{i=1}^{T_{\mathrm{tat}}}\left(\mathbf{1}_{\mathrm{GL}}(t) c_{\mathrm{GL}}(t)+\sum_{i=1}^{N}\left(\mathbf{1}_{\mathrm{CM} i}(t) c_{\mathrm{CM} i}+\mathbf{1}_{\mathrm{PM} i}(t) c_{\mathrm{PM} i}+\mathbf{1}_{\mathrm{OM} i}(t) c_{\mathrm{OM} i}\right)\right)
$$

where $c_{\mathrm{CM} i}, c_{\mathrm{PM} i}, c_{\mathrm{OM} i}$ are defined as each corrective maintenance cost, preventive maintenance cost and opportunistic maintenance for component $i$, respectively. $c_{\mathrm{GL}}(t)$ is defined as the generation loss during the period $t$. Meanwhile, $\mathbf{1}_{\mathrm{CM} i}(t), \mathbf{1}_{\mathrm{PM} i}(t), \mathbf{1}_{\mathrm{OM} i}(t)$ are equal to one if corrective maintenance, preventive maintenance or opportunistic maintenance occurs to component $\mathrm{i}$ during period $\mathrm{t}$, respectively, and they are equal to zero for other cases. $\mathbf{1}_{\mathrm{GL}}(t)$ is equal to one if the multi-component system is down for the maintenance actions during period $\mathrm{t}$, and it is equal to zero for other cases. The objective of the proposed policy is to find the optimal risk threshold scaling factor values $\left\{y_{1}, y_{2}\right\}$ to minimize the expected maintenance cost per unit time during the planning horizon. The optimization model can be formulated as follows:

$$
\begin{aligned}
& \min C\left(y_{1}, y_{2}\right) \\
& \text { s.t. } y_{1}>y_{2}>0 .
\end{aligned}
$$

A numerical algorithm was developed for the cost evaluation of a PHM based CBM with respect to determined risk thresholds, more detail can be seen in the Ref. [14]. However, this kind of cost evaluation method is time-consuming as the component number and covariate number increase, since the degradation of the generating unit is stochastic process. To balance the computation time and computational accuracy, the cost evaluation method based on Monte Carlo simulation [19] method is applied to calculate the expected maintenance cost during the planning horizon, and then the optimal thresholds can be obtained by minimizing the expected maintenance cost. 


\section{Examples}

In this section, long-term monthly inflow data covering January 1942 to December 2012 of Xiao Dongjiang River Hydro-plant station in the Dongjiang River basin are analyzed, as show in Figure 1. The hydro plant is run-off-river plant, and it has no water storage. So the monthly penstock releases $Q(t)$ is equal to the inflows if the inflow is among the range of design flow, i.e., $Q(t)=R(t)$. For this power plant, the upper level $Z_{1}$ is 148.0 meters, and the tail water level $Z_{2}$ can be obtained from the relationship between the discharge-tail water levels, i.e., $Z_{2}(t)=f(Q(t))$, as shown in Figure 2, by linear interpolation. So the head can be calculated by $H(t)=Z_{1}-Z_{2}(t)$.

Then the monthly generation output can be calculated by $N(t)=k Q(t) H(t)$, where $k$ is defined as the output factor and $k$ is equal to 6.0 in this case. Then the generation losses during each maintenance duration can be calculated to be $C_{\mathrm{GL}}=N T_{\mathrm{D}} U$, where $T_{\mathrm{D}}$ is defined as maintenance duration and is set to be 24 hours in this research, and $U$ is defined as the monthly average electricity price and is set to be $52 \$ / \mathrm{Mwh}$. The average electricity price is analyzed from the PJM power market [20].

The hydro generating unit can be treated as a series system connected by hydro turbine, generator and transformer. A failure of any component will lead to system malfunction, so there is economic dependence among the components. We choose condition indicator as covariate. Condition indicator (CI) is quantitative rating given after assessment of equipment thoroughly [21]. The condition indicator varies from 0 to 100 , and scoring is done with respect to the present physical state of the component. Based on the failure, maintenance events and concomitant condition indicators of the three critical components of a hydro generating unit, the PHM parameters can be thus estimated [22], and the parameters for the components are given in Table 1.

The transition probability matrix for critical components (Table 2, 3 and 4) is required to calculate the cost evaluation of the proposed policy. The transition probability matrix gives the possibilities of a covariate going from current range to the range at the next inspection time. Assume the inspection interval is 30 days. The transition probability matrix can be estimated by the history information for condition indicators of different components. Covariate ranges are defined for four levels, such as between 0 and 35, between 35 and 60 , between 60 and 85 , between 85 and 100. They are selected by combining practical experience and covariate distribution histograms. These four ranges are referred to as four states: state 1, 2,3 and 4 . Thus the highest possible component state is $J=4$, and a component is in state 1 if the condition indictor falls into range $[0,35)$ and so on.

The corrective maintenance cost, preventive maintenance cost for critical components of the hydro generating unit is presented in Table 5. The time-dependent inflow and the contrast of constant and inflow-dependent thresholds are shown in Figure 3. In this research the inflow of the hydro plant is determined instead of stochastic. The case with stochastic inflows will be discussed in future work. The cost of generation loss if the generating unit is down for each period is time dependent since the long-term inflow is varying. The planning horizon $T_{\text {tal }}$ is 48 months in this case. 


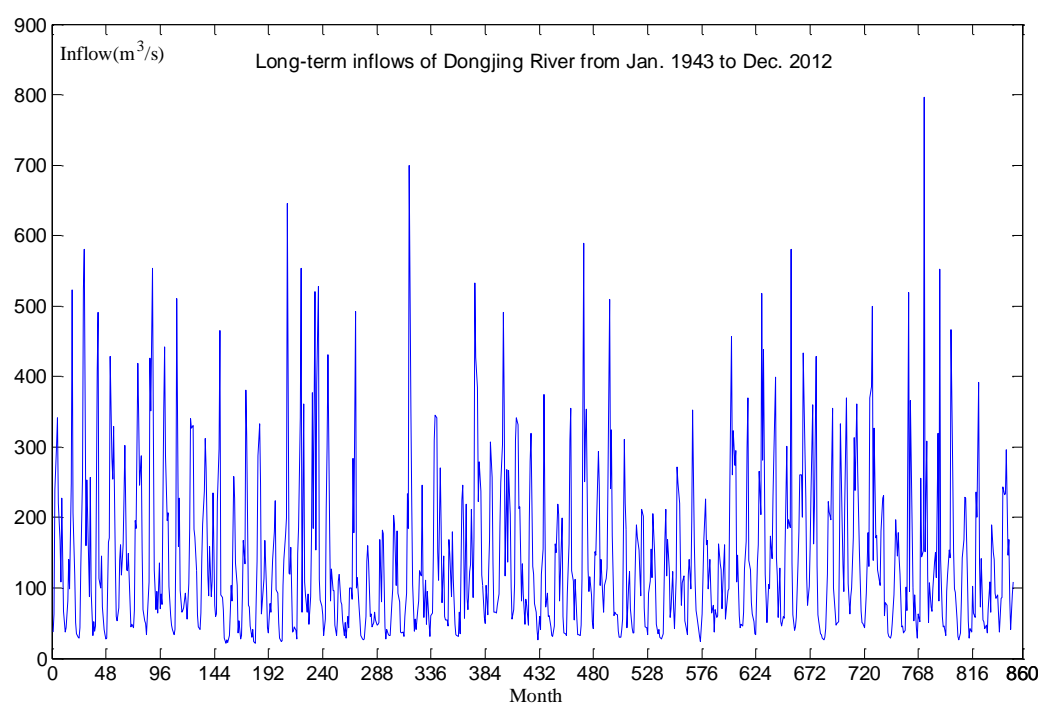

Figure 1. Long-term history inflows of Dongjiang River from Jan. 1943 to Dec. 2012

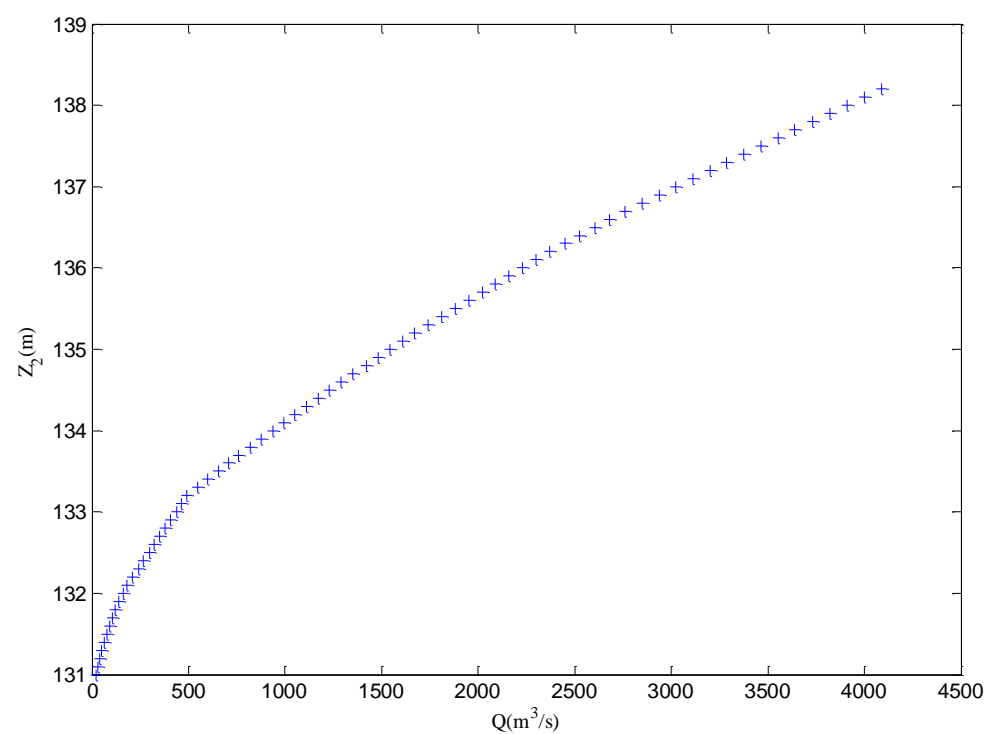

Figure 2. Curve of Tail Water Level $Z_{2}$ Versus Discharge $Q$

Table 1. Parameters of Proportional Hazards Model for Critical Components

\begin{tabular}{cccc}
\hline Component & Shape Parameter $\beta$ & Scaling Parameter $\eta$ & Coefficient parameter $\gamma$ \\
\hline Hydro turbine & 3 & 1000 & 0.061 \\
Generator & 2 & 1250 & 0.044 \\
Transformer & 3 & 800 & 0.026 \\
\hline
\end{tabular}


Table 2. Transition Probability Matrix of Condition Indictor of Hydro Turbine

\begin{tabular}{ccccc}
\hline Bands & {$[\mathbf{0 ,}, \mathbf{3 5})$} & $\mathbf{[ 3 5 , 6 0 )}$ & {$[\mathbf{6 0 , 8 5})$} & {$[\mathbf{8 5 , 1 0 0 )}$} \\
\hline$[0,35)$ & 0.72350 & 0.25340 & 0.02258 & 0.00052 \\
{$[35,60)$} & 0.03301 & 0.85120 & 0.11490 & 0.00089 \\
{$[60,85)$} & 0.01800 & 0.19220 & 0.78710 & 0.00270 \\
{$[85,100)$} & 0 & 0 & 0 & 1 \\
\hline
\end{tabular}

Table 3. Transition Probability Matrix of Condition Indictor of Generator

\begin{tabular}{ccccc}
\hline Bands & {$[\mathbf{0 ,}, \mathbf{3 5})$} & {$[\mathbf{3 5 , 6 0})$} & {$[\mathbf{6 0 , 8 5})$} & {$[\mathbf{8 5 , 1 0 0 )}$} \\
\hline$[0,35)$ & 0.79850 & 0.18180 & 0.01921 & 0.00049 \\
{$[35,60)$} & 0.02815 & 0.83270 & 0.13840 & 0.00075 \\
{$[60,85)$} & 0.02110 & 0.12250 & 0.74320 & 0.11320 \\
{$[85,100)$} & 0 & 0 & 0 & 1 \\
\hline
\end{tabular}

Table 4. Transition Probability Matrix of Condition Indictor of Transformer

\begin{tabular}{ccccc}
\hline Bands & {$[\mathbf{0 ,}, \mathbf{3 5})$} & $\mathbf{[ 3 5 , 6 0 )}$ & $\mathbf{[ 6 0 , 8 5})$ & {$[\mathbf{8 5 , 1 0 0 )}$} \\
\hline$[0,35)$ & 0.73590 & 0.23310 & 0.03038 & 0.00062 \\
{$[35,60)$} & 0.00926 & 0.82920 & 0.16070 & 0.00084 \\
{$[60,85)$} & 0.00794 & 0.09850 & 0.81030 & 0.08326 \\
{$[85,100)$} & 0 & 0 & 0 & 1 \\
\hline
\end{tabular}

Table 5. Corrective and Preventive Maintenance Cost for Major Components

\begin{tabular}{|c|c|c|c|c|c|c|c|c|}
\hline Component & \multicolumn{4}{|c|}{ Corrective maintenance cost $(\$ 1000)$} & \multicolumn{4}{|c|}{ Preventive maintenance cost $(\$ 1000)$} \\
\hline Hydro turbine & \multicolumn{4}{|c|}{71} & \multicolumn{4}{|c|}{12} \\
\hline Generator & \multicolumn{4}{|c|}{50} & \multicolumn{4}{|c|}{10} \\
\hline Transformer & \multicolumn{4}{|c|}{70} & \multicolumn{4}{|c|}{12} \\
\hline \multicolumn{9}{|c|}{$\begin{array}{l}\text { Table 6. Comparative Result for Proposed Policy Compared to Constant } \\
\text { Control-Limit Policy }\end{array}$} \\
\hline policy & $\begin{array}{c}x_{1} \text { or } y_{1} \\
\left(\log _{10}\right)\end{array}$ & $\begin{array}{c}x_{2} \text { or } y_{2} \\
\left(\log _{10}\right)\end{array}$ & Outages & $\mathrm{CM}$ & PM & $\mathrm{OM}$ & $\begin{array}{c}\text { cost } \\
(\$ / \text { day })\end{array}$ & cost-saving \\
\hline $\begin{array}{l}\text { Constant } \\
\text { threshold policy }\end{array}$ & -1 & -1.1 & 12.7 & 3.1 & 11.3 & 3.1 & 390 & \multirow{2}{*}{$8 \%$} \\
\hline $\begin{array}{l}\text { Inflow-dependent } \\
\text { threshold policy }\end{array}$ & -1 & -1.1 & 12.8 & 2.8 & 12.4 & 1.6 & 360 & \\
\hline
\end{tabular}

We perform a comparative study between the constant threshold and inflow-dependent threshold policy for the hydroelectricity generating unit. The cost of the constant control-limit policy can be evaluated as threshold values $x_{1}$ and $x_{2}$ are determined. Meanwhile the cost of inflow-dependent control-limit CBM policy can also be evaluated through certain risk threshold scaling factor values $y_{1}$ and $y_{2}$. With respect to the two kinds of CBM policy, the optimal cost values and the optimal CBM policies are listed in Table 6. We can see that the optimal cost of inflow-dependent threshold policy is about $8 \%$ lower than that of the constant 
threshold policy. For detailed analysis as shown in Table 7, we compare the two kinds of CBM policies with: 1) the average number of outages of the generating unit, 2) the average number of corrective maintenances for all the components, 3) the average number of corrective maintenances for all the components, 4) the average number of opportunistic maintenances for all the components. It can be seen that the average number corrective maintenance (i.e., failures) was reduced from 3.1 to 2.8 and the average number of preventive maintenance increases from 11.3 to 12.4. Meanwhile the average number of outages is similar to that of the constant threshold policy.
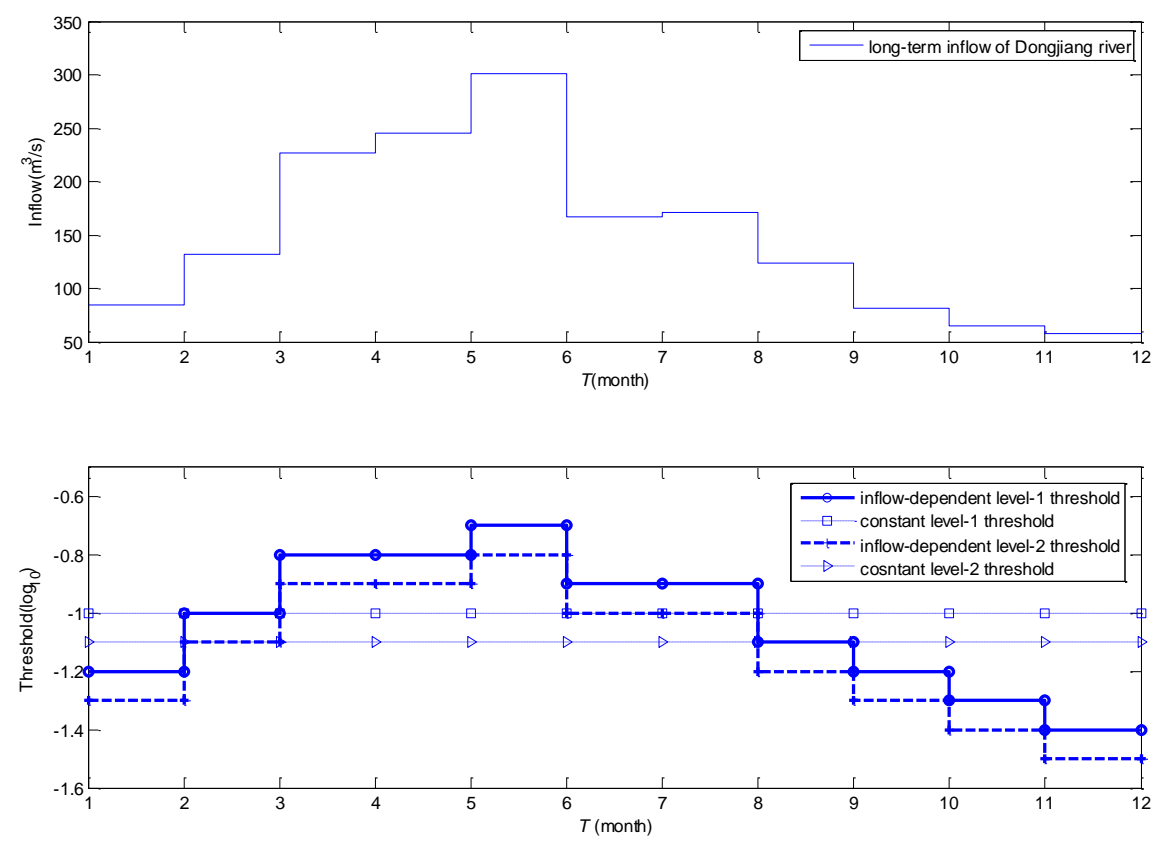

\section{Figure 3. The Long-term Inflow of the Dongjiang River and the Comparison between Inflow-Dependent Threshold and the Constant Threshold Policy}

The reason for the cost-effectiveness of the proposed policy is that preventive maintenance can be performed with much higher possibilities during the low-inflow periods while preventive maintenance can be performed with much lower possibilities during the highinflow periods. More details can be seen from the comparative study of event possibilities between constant threshold and inflow-dependent threshold policy Figure 3. In comparative to the constant threshold policy, the Figure 4 shows that the possibilities of preventive maintenance are much higher for high-inflow periods, and are much lower for low-inflow periods. As a result, compared to the constant threshold policy, the possibilities of failures (i.e., corrective maintenance) are a little higher during high-inflow periods and are much lower during low-inflow periods. In other words, the possibilities of PMs are much higher during low-inflow periods than that during the high-inflow periods. As a result, the possibilities of failures (i.e., corrective maintenance) are much higher during high-inflow periods than that during low-inflow periods. In other words, during higher-inflow periods failure can occur with a little higher possibility since preventive maintenance is less likely to be performed. However, the objection function value (i.e., maintenance cost rate) of the 
inflow-dependent threshold policy can be reduced significantly since the preventive maintenance actions are more than the failures. That is to say, the increasing cost by performing corrective maintenance during the high-inflow periods is less than the cost-saving by performing preventive maintenance more likely during the low-inflow periods.

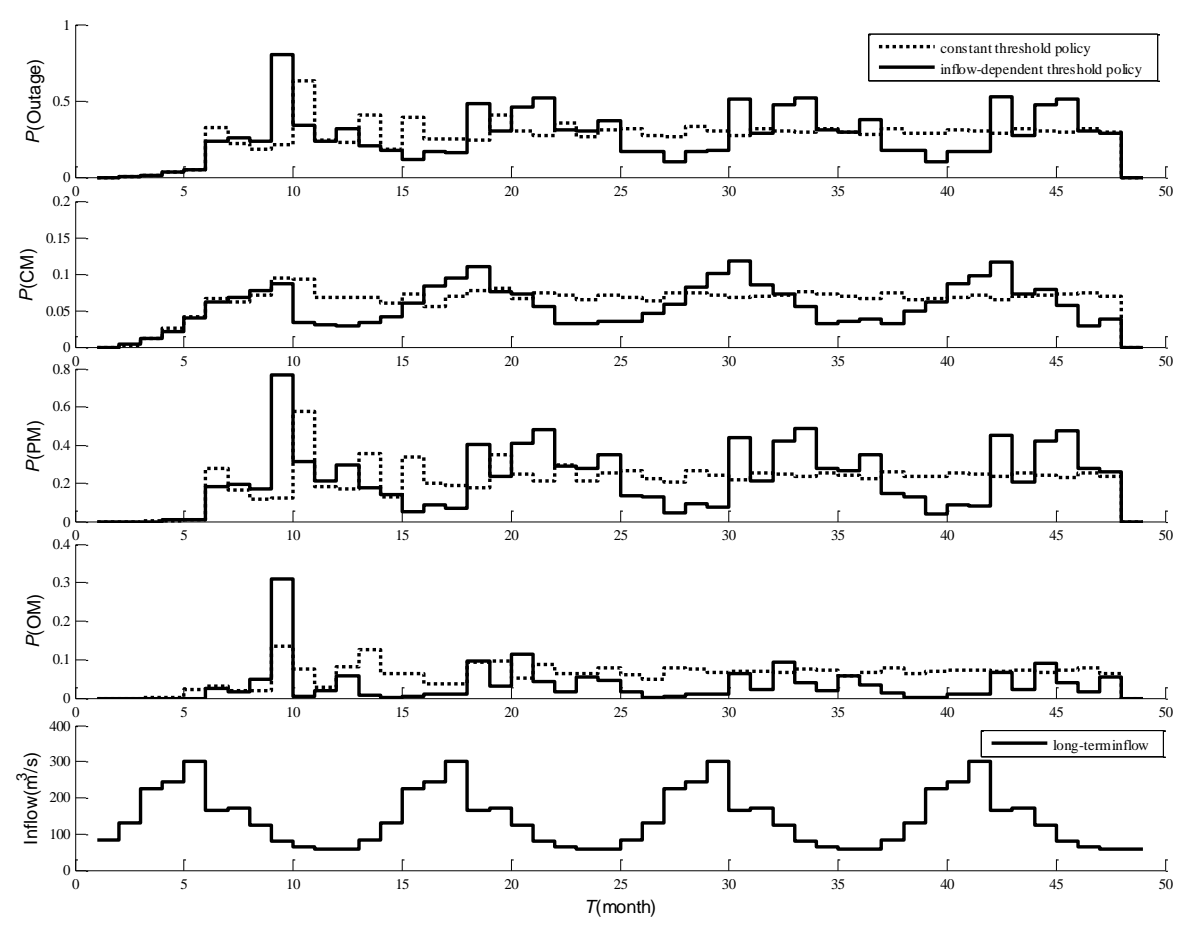

Figure 4. The Event Possibilities for the Inflow-dependent Threshold and the Constant Threshold Policy

\section{Conclusion}

In this research, we have proposed the inflow-dependent control-limit policy for condition based maintenance optimization for the hydro-electricity generating unit considering the timedependent economic dependence among components. For the proposed CBM policy, the risk threshold is dependent on the time-varying inflows instead of being constant value. So the inflow-dependent threshold is represented by the product of the time-dependent inflow and the risk threshold scaling factors for the proposed CBM policy. Since the cost evaluation for proposed CBM policy becomes much more complex when the number of components becomes large and the types of the components are different, Monte Carlo simulation method is applied for the proposed policy to efficiently reduce the computation time. From the simulation cases, it shows that the cost-effectiveness of the proposed policy since the costsaving is significant compared to the constant threshold policy. Future research topics will be to develop CBM policies for the multi-component system with stochastic inflows and uncertainties. 


\section{References}

[1] M. Munasinghe, W.G. Scott and M. Gellerson, "Economics of power system reliability and planning", Theory and case study, International Bank for Reconstruction and Development, Washington, DC (USA), (1979).

[2] P. K. Koner and G. Ledwich, "Distribution Network Optimisation for Enhanced Reliability", Journal of Electrical \& Electronics Engineering, Australia, vol. 21, no. 3, (2002), pp. 199-208.

[3] R. Billinton, M. Fotuhi-Firuzabad and L. Bertling, "Bibliography on the application of probability methods in power system reliability evaluation 1996-1999”, Power Systems, IEEE Transactions on, vol. 16, no. 4, (2001), pp. 595-602.

[4] H. Singh and A. Papalexopoulos, "Competitive procurement of ancillary services by an independent system operator", Power Systems, IEEE Transactions on, vol. 14, no. 2, (1999), pp. 498-504.

[5] R. Mukerji, H. M. Merrill, B. W. Erickson, J. H. Parker and R. E. Friedman, "Power plant maintenance scheduling: optimizing economics and reliability", Power Systems, IEEE Transactions on, vol. 6, no. 2, (1991), pp. 476-483.

[6] H. Kim, Y. Hayashi and K. Nara, "An algorithm for thermal unit maintenance scheduling through combined use of GA, SA and TS", Power Systems, IEEE Transactions on, vol. 12, no. 1, (1997), pp. 329-335.

[7] L. Thomas, "A survey of maintenance and replacement models for maintainability and reliability of multiitem systems", Reliability Engineering, vol. 16, no. 4, (1986), pp. 297-309.

[8] H. Wang, "A survey of maintenance policies of deteriorating systems", European Journal of Operational Research", vol. 139, no. 3, (2002), pp. 469-489.

[9] M. Berg and B. Epstein, "Comparison of age, block, and failure replacement policies", Reliability, IEEE Transactions on, vol. 27, no. 1, (1978), pp. 25-29.

[10] D. Cho and M. Parlar, "A survey of maintenance models for multi-unit systems", European Journal of Operational Research, vol. 51, no. 1, (1991), pp. 1-23.

[11] X. Zheng, and N. Fard, "A maintenance policy for repairable systems based on opportunistic failure-rate tolerance”, Reliability, IEEE Transactions on, vol. 40, no. 2, (1991), pp. 237-244.

[12] R. Dekker, R. E. Wildeman and F. A. van der Duyn Schouten, " A review of multi-component maintenance models with economic dependence", Mathematical Methods of Operations Research, vol. 45, no. 3, (1997), pp. 411-435.

[13] A. Heng, S. Zhang, A. C. C. Tan and J. Mathew, "Rotating machinery prognostics: State of the art, challenges and opportunities", Mechanical Systems and Signal Processing, vol. 23, no. 3, (2009), pp. 724739.

[14] Z. Tian and H. Liao, "Condition based maintenance optimization for multi-component systems using proportional hazards model”, Reliability Engineering \& System Safety, vol. 96, no. 5, (2011), pp. 581-589.

[15] A. K. S. Jardine, D. M. Lin and D. Banjevic, "A review on machinery diagnostics and prognostics implementing condition-based maintenance", Mechanical Systems and Signal Processing, vol. 20, no. 7, (2006), pp. 1483-1510.

[16] A. K. S. Jardine, V. Makis, D. Banjevic, D. Braticevic and M. Ennis, "A decision optimization model for condition-based maintenance", Journal of Quality in Maintenance Engineering, vol. 4, no. 2, (1998), pp. 115121.

[17] A. K. S. Jardine, D. Banjevic, D. W. Wiseman, S. Buck and T. Joseph, "Optimizing a mine haul truck wheel motors' condition monitoring program Use of proportional hazards modeling”, Journal of Quality in Maintenance Engineering, vol. 7, no. 4, (2001), pp. 286-302.

[18] D. Banjevic, A. K. S. J. V. Makis and M. Ennis, "A control-limit policy and software for condition-based maintenance optimization”, INFOR, vol. 39, no. 1, (2001), pp. 32.

[19] M. Marseguerra, E. Zio and L. Podofillini, "Condition-based maintenance optimization by means of genetic algorithms and Monte Carlo simulation”, Reliability Engineering \& System Safety, vol. 77, no. 2, (2002), pp. 151-165.

[20] Monthly Locational Marginal Pricing. Available from: http://www.pjm.com/markets-andoperations/energy/real-time/monthlylmp.aspx.

[21] S. R. Sharma, "Feasibility study of options for aging hydro-power generation facility", Master Thesis, The University of Texas at Arlington, (2006).

[22] D. Percy and B. Alkali, "Generalized proportional intensities models for repairable systems", IMA Journal of Management Mathematics, vol. 17, no. 2, (2006), pp. 171-185. 


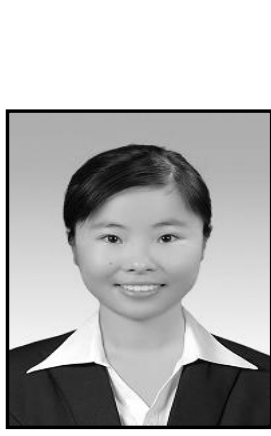

\section{Authors}

Xinbo Qian, she was born in Jiangsu province, China on January 10, 1986. She received her bachelor degree in Thermal and Power Engineering from Hohai University, Nanjing in 2008. Presently she is a $\mathrm{PhD}$ candidate from School of Hydropower and Information Engineering, Huazhong University of Science and Technology, Wuhan. Her current research is related to the condition-based maintenance of hydroelectric power plants in the context of electricity markets.

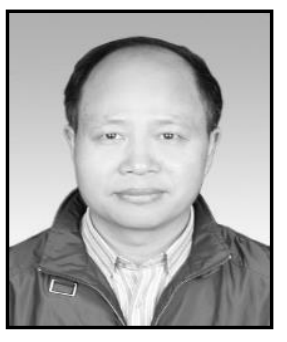

Yonggang Wu, he was born in Hunan province, China on October 27, 1963. He received his B.S. degree in automatic control engineering in 1984 and M.S. degree in electrical engineering in 1987, all from Huazhong University of Science and Technology (HUST). He received the degree of Ph.D. in power systems and automation in 1997, also from HUST. Since 1987, he has been researching in power system and plant optimization and control, hydro-thermal electrical planning. Now he is a full professor of the School of Hydropower and Information Engineering, HUST. 
International Journal of Security and Its Applications Vol.8, No.2 (2014) 